\title{
Editorial Overview: Hollow and Porous Materials for Lithium-Ion Battery Anode
}

\author{
Manab Kundu* \\ Department of Material Science and Engineering, Norwegian University of Science and Technology, Norway
}

Submission: July 24, 2017; Published: August 03, 2017

*Corresponding author: Manab Kundu, Department of Material Science and Engineering, Norwegian University of Science and Technology, (NTNU), NO-7491 Trondheim, Norway, Tel: +47 735 51218; E-mail: manab.kundu@ntnu.no, chemmanab@gmail.com

Keywords: Lithium-ion battery; Energy density; Power density; Microstructure

Abbrevations: LIB: Lithium-Ion Battery; HED: High Energy Density; HPD: High Power Density

\section{Editorial Overview}

Lithium-ion battery (LIB) have become most important power sources of today's portable electronic devices and electric/hybrid vehicles due to their superior volumetric and gravimetric energy densities. However, the gradually growing power requirement creates a huge doubt that if LIB will be able to meet this demand as portable energy storage in the long-term run. Hence, the next generation LIB should be accomplished with few key features: high capacity to last longer (high energy density), fast charging process (high power density), long life, safety and low cost.

In order to increase the capacity of LIB, researcher needs to find suitable anode and cathode materials with high specific capacity. As high capacity anode material, although various transition/non-transition metal oxides (TMOs), silicon and Germanium anodes have been studied extensively [1], still only graphitic carbon is the only commercially used anode. In maximum reports, authors generally highlight the capacity values of the non-graphitic anodes, but key issue like de-lithiation potential need to be considered. Unlike anode, in LIB many cathodes are already been commercialized: LiCO204, LiFePO4, LiNi0.8Co0.15Al0.05, LiNi1-x-yMnxCoy etc [2]. Even though, significant progress in LIB electrode has been made, however, great efforts are still needed. Specifically, researcher needs to find a suitable alternative anode material having not only high capacity, but with reasonably lower de-lithiation potential.

On the other hand, the demand for faster charging process i.e. improving the rate capability of the LIBs, there are still more rooms for improving the current electrode materials to deliver faster performance. There are several factors, which limit the rate capability of LIB electrode materials [3].

i. Electrical Conductivity: Most of the LIB electrode materials suffer from poor electrical conductivity which affects the electron transfer between the redox sites and the current collector. The most common solutions are to utilize a conductive agent to improve the conductivity between the electro active particles and modifying the interfacial connection by introducing a thin layer of graphene coating on current collector.

ii. Solid state diffusion: The solid state diffusion of lithium ions within the bulk electrode material is the rate limiting step in a lithium-ion battery. Hence, the morphology and micro-structure of electrode material has a huge impact on the rate capability.

In order to significantly improve the electro chemical performance, various electrode materials with different nanostructures and morphologies have been studied such as nanoparticles, nano wires, nano sheets, nano rods, microspheres and so on [4]. Many researchers also argued the advantageous side of binder free electrode materials where the electrodes are grown directly on the current collector without using any binder and conductive agent. These binder free electrodes exhibit many inherent advantages over the conventional slurry-cast electrode with respect to capacity as well as rate capability [5].

i. The shortened transport length for $\mathrm{Li}+$ ion diffusion inside the electrode which accelerates the mass transfer kinetics. 
ii. The high contact surface area between electrolyte and active material makes $\mathrm{Li}+$ ions more accessible to the active materials and enhances the electrochemical reaction kinetics.

iii. Less interface transfer resistance allows rapid electron collection and transportation to outer circuit. Unfortunately, the poor loading mass of these binder free electrodes reduces the gravimetric as well as volumetric capacity of the final device significantly- the main obstacle for commercial application.

Hollow nanoparticles with porous outer shell and having a low void volume, might be the ideal structure for an electrode material in LIBs because of their structural stability and high volumetric energy density. The hollow interior morphology create a smooth pathway for $\mathrm{Li}+$ and electron diffusion, and in the same time, the empty space act as a reservoir for electrolyte to decrease electrolyte diffusion resistance during the lithiation and de lithiation processes[6]. Moreover, the mesoporous shell not only provide a larger surface area towards $\mathrm{Li}+$ and electrolyte, directs the volume expansion towards the inner hollow core during the lithiation, and during the de lithiation, the morphology recovers. Thus, the hollow spheres can effectively alleviate the volume changes during the lithiation/ de-lithiation processes for cycling stability [7]. In addition, the hollow nanostructure could facilitate the electrolyte infiltration for faster electrochemical reaction during the repeated charge discharge process. Therefore, it's time for researcher to find and develop a novel, simple and cost effective process which would be commercially viable for hollow nano spheres synthesis.

\section{References}

1. Tang T, Hou Y (2016) Nano structured Anode Materials for Lithium Ion Batteries: Progress, Challenge and Perspective Nasir Mahmood. Adv Energy Mater 1600374.

2. Blomgren GE (2017) The Development and Future of Lithium Ion Batteries. J Electro chem Society 164(1): A5019-A5025.

3. Eftekhari A (2017) Lithium-Ion Batteries with High Rate Capabilities. ACS Sustainable Chem Eng 5(4): 2799-2816.

4. Fan B, Chen X, Hu A, Tang Q Fan H, et al. (2016) facile synthesis of 3D plum candy-like $\mathrm{ZnCO}_{2} \mathrm{O}_{4}$ microspheres as a high-performance anode for lithium ion batteries. RSC Adv 6: 79971-79977.

5. Feng Z, Qi L (2016) Recent Progress in Self-Supported Metal Oxide Nanoarray Electrodes for Advanced Lithium-Ion Batteries. Adv Sci 1600049.

6. Kundu M, Karunakaran G, Kolesnikov E, Dmitry A, Gorshenkov M V, et al. (2017) Hollow (Co0.62 $\mathrm{Fe} 1.38$ ) $\mathrm{FeO}_{4} / \mathrm{NiCO}_{2} \mathrm{O}_{4}$ nanoboxes with porous shell synthesized via chemical precipitation: A novel form as a high performance lithium ion battery anode. Microporous Mesoporous Mater 247(2017): 9-15.

7. Cho JS, Hong YJ, Lee JH, Kang YC (2015) Design and synthesis of micronsized spherical aggregates composed of hollow $\mathrm{Fe}_{2} \mathrm{O}_{3}$ nanospheres for use in lithium-ion batteries. Nanoscale 7(18): 8361-8367.

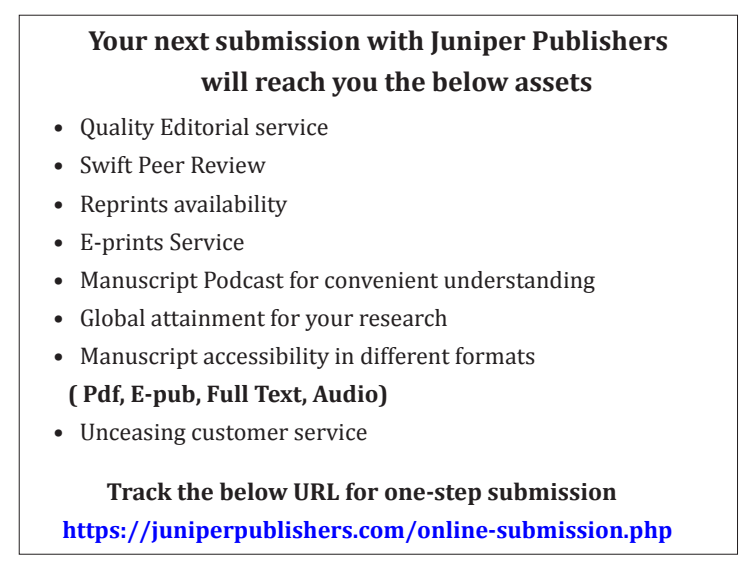

